\title{
Peierls-Nabarro model of interfacial misfit dislocation: An analytic solution
}

\author{
Yugui Yao* and Tsuchiang Wang \\ LNM, Institute of Mechanics, Chinese Academy of Sciences, Beijing 100080, China \\ Chongyu Wang \\ Central Iron and Steel Research Institute, Beijing 100081, China \\ (Received 14 August 1998; revised manuscript received 30 October 1998)
}

\begin{abstract}
We propose a method to treat the interfacial misfit dislocation array following the original Peierls-Nabarro's ideas. A simple and exact analytic solution is derived in the extended Peierls-Nabarro's model, and this solution reflects the core structure and the energy of misfit dislocation, which depend on misfit and bond strength. We also find that only with $\beta<0.2$ the structure of interface can be represented by an array of singular Volterra dislocations, which conforms to those of atomic simulation. Interfacial energy and adhesive work can be estimated by inputting ab initio calculation data into the model, and this shows the method can provide a correlation between the $a b$ initio calculations and elastic continuum theory.

[S0163-1829(99)00512-3]
\end{abstract}

\section{INTRODUCTION}

The importance of interfaces between dissimilar materials cannot be emphasized enough in many areas, such as in heterostructure devices, metal-ceramic composites, and protective coatings. Interfaces play crucial roles in determining the properties and performance of materials. They may be completely incoherent, coherent but strained or semicoherent. The last case is characterized by the presence of misfit dislocations (MD). These dislocations at interface are geometrically necessary defects, which are part of the interfacial structure, and they reduce the misfit strain with respect to the unrelaxed fully coherent interface by locally decreasing the interfacial coherency. ${ }^{1}$

The concept of misfit dislocation was introduced by Frank and Van der Merwe ${ }^{2}$ in a Frenkel-Kontorva-type model for the oriented monolayer overgrowths in 1949. Since then, there have been numerous observations of misfit dislocations in epitaxial thin films as reviewed by Matthews, ${ }^{3}$ and some in metal-ceramic composites, ${ }^{4,5}$ On the theoretical part, the studies were carried out almost all by the continuum theory of dislocation and mainly focused on transformation of coherently grown epitaxial films into semicoherent ones as the thickness of the film increasing, ${ }^{1,6}$ and very limited atomic calculations were performed for metal-metal interfaces and metal-ceramic interfaces. $5,7,8$

As we all know, the continuum theory which does not involve any atomic level information cannot response the core structure of misfit dislocation. On the other hand, atomic simulations can reflect atomic level information, while it takes tedious and intensive computational processes. The Peierls-Nabarro (PN) model, ${ }^{9}$ though relatively simple, can bridge the continuum theory and atomic theory, and has the potential of providing quantitative estimation for atomistic property of the dislocations. Recently, the PN model has been extended to estimate Peierls stress directly from the generalized stacking energy surface, for both narrow and wide dislocations. ${ }^{10}$ Merwe $^{11,12}$ had studied interfacial MD by the Fourier analysis procedure (we call it Merwe's method in the context). However, Merwe's method is mathematically complex, and until now, the exact analytic solution have not yet been obtained for the PN model when the components of interface have different elastic parameters. ${ }^{12}$ In this paper, we take an alternative method, which follows the original Perierls-Nabarro's idea and is simpler than Merwe's, to deal with the similar problem. By means of this method, we derive an exact analytic solution, i.e., a concise expression of displacement field of misfit dislocation. In the discussion, we focus on two questions: one is how the MD core energy and structure depend on both misfit and the bond strength parallel to the interface; and the other is how to input $a b$ initio calculation data into our model.

\section{PEIERLS-NABARRO MODEL}

We consider a two-layer, which is illustrated in Fig. 1, composed of the two cubic crystals, 1 (upper) and 2 (lower) joined at $\{001\}$ interface, and MD array is positioned at the interface. We make the conventional assumptions: (i) the crystals 1 and 2 have lattice parameters $a_{1}$ and $a_{2}$, respectively $\left(a_{1}>a_{2}\right)$, (ii) $a_{1}$ and $a_{2}$ may be generated from a reference lattice with parameter $c$ defined by ${ }^{13}$

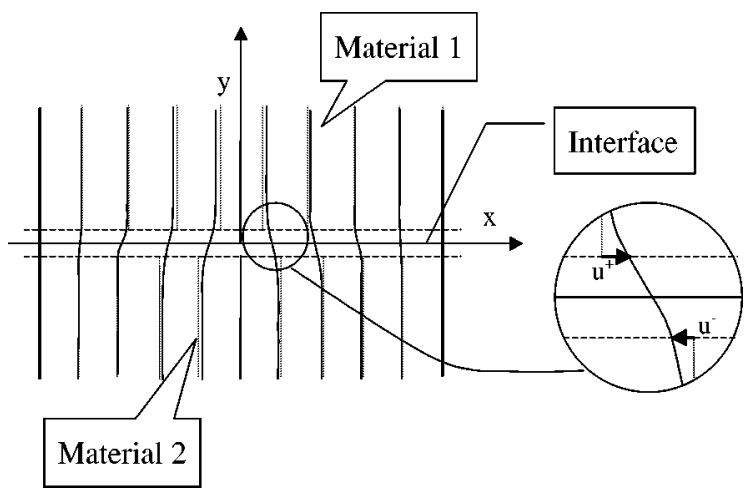

FIG. 1. Misfit-edge dislocation at the interface. 


$$
p=P a_{1}=(P+1) a_{2}=\left(P+\frac{1}{2}\right) c,
$$

where $P$ is an integer, and (iii) the crystals deform under applied forces like isotropic elastic continua with shear moduli $\mu_{1}$ and $\mu_{2}$ and Poisson's ratios $\nu_{1}$ and $\nu_{2}$, respectively. Equation (1) defines misfit $f, c$, and the MD spacing $p$ as

$$
\begin{gathered}
p=\frac{a_{1} a_{2}}{a_{1}-a_{2}} \\
c=\frac{2 a_{1} a_{2}}{\left(a_{1}+a_{2}\right)} \\
f=\frac{c}{p}=\frac{2\left(a_{1}-a_{2}\right)}{\left(a_{1}+a_{2}\right) .}
\end{gathered}
$$

As shown in Fig. 1, we assume the dislocation core to be confined within $x 0 z$ glide plane (i.e., the interface), and the Burgers vector $\vec{b}$ to be parallel to the $x$ axis and the dislocation line to be chosen as the $z$-axis direction. For singleedge dislocation at interface, using the result of continuum theory, the relevant shear stress at interface is ${ }^{14}$

$$
\sigma_{x y}=\frac{2 \mu_{2}}{\left(\kappa_{2}+1\right)} \frac{\left(1+\alpha_{1}\right)}{\left(1-\alpha_{2}^{2}\right) \pi}\left(\frac{b}{x}\right) .
$$

Here, $\alpha_{1}, \alpha_{2}$ are Dundurs' parameters. ${ }^{14}$ For the periodic dislocation array in Fig. 1, the relevant shear stress along interface is

$$
\sigma_{x y}=\frac{2 \mu_{2}}{\left(\kappa_{2}+1\right)} \frac{\left(1+\alpha_{1}\right) b}{\left(1-\alpha_{2}^{2}\right) \pi} \sum_{n=-\infty}^{\infty} \frac{1}{x+n p} .
$$

Using identities $\Sigma_{-\infty}^{+\infty} 1 / n+a=\pi \cot \pi a,{ }^{15}$ one obtains

$$
\sigma_{x y}=\frac{2 \mu_{2}}{\left(\kappa_{2}+1\right)} \frac{\left(1+\alpha_{1}\right) b}{\left(1-\alpha_{2}^{2}\right) p} \cot \frac{\pi x}{p} .
$$

Following the original PN model, ${ }^{9}$ within the glide plane, at each point with a distance $x^{\prime}$ from the dislocation line, the plastic displacement $u\left(=u^{+}-u^{-}\right)$of the upper part of the interface $(y>0)$ with respect to the lower part $(y<0)$ results from the continuous distributions of infinitesimal dislocations with the Burgers vector density $\rho\left(x^{\prime}\right) d x^{\prime}=$ $-\left[d u\left(x^{\prime}\right) / d x^{\prime}\right] d x^{\prime}$. The component along $\vec{b}$ of the total resultant stress at $x, \sigma_{x y}(x)$, is the sum of the contributions from all these infinitesimal dislocations. $\sigma_{x y}(x)$ is balanced by the corresponding component of the periodic restoring force stress $F[U(x)]$ acting between atoms on either side of the interface. $U(x)$, composed of plastic displacement and misfit displacement, is the relative displacement between corresponding atoms on either side,

$$
U(x)=\frac{c}{2}+\frac{c}{p} x+u(x) .
$$

For the case of periodic MD array at interface, using Eq. (5) we can obtain the following integro-differential equation similar to $\mathrm{PN}$ equation

$$
-\mu \int_{-p / 2}^{p / 2} \frac{1}{p} \cot \left[\frac{\pi\left(x-x^{\prime}\right)}{p}\right] \frac{d u\left(x^{\prime}\right)}{d x^{\prime}} d x^{\prime}=F[U(x)],
$$

with the boundary conditions $u(-p / 2)=0$ and $u(p / 2)$ $=0$. $\mu$ depends on the elastic constants of two materials, and equals to $\left\{\left[2 \mu_{2} /\left(\kappa_{2}+1\right)\right]\left[\left(1+\alpha_{1}\right) /\left(1-\alpha_{2}^{2}\right)\right]\right\}$. Similar to the original PN model, the restoring stress $F(U)$ assumes a sinusoidal form

$$
F[U(x)]=\frac{\tau}{2 \pi} \sin [2 \pi U(x) / c]
$$

and $\tau$ is defined as bond strength parallel to the interface. This sinusoidal approximation may, however, be very crude. A more physical interpretation of the restoring stress $F(u)$ is to consider it as the gradient of the so-called generalized stacking fault energy ( $g s f$ or $\gamma$ ) (Ref. 16) surface, which can be obtained by $a b$ initio calculation, ${ }^{17}$ in the appropriate direction for the dislocation under study. Then the restoring stress is simply $F(u)=-\partial \gamma / \partial u .^{18}$ In the case of interface, the $g s f$ surface $\gamma(u)$ (energy per unit area) can be generated from the energy of the displaced interface in a reference lattice with parameter $c$ defined by Eq. (2), as material 1 displaces with respect to material 2 at interface. Interfacial $g s f$ obtained by $a b$ initio calculation will be given in detail in other paper. In this paper, we still assume sinusoidal restoring force.

With the help of Eqs. (6) and (8), Eq. (7) can be transformed into

$$
\begin{aligned}
& -\mu \int_{-p / 2}^{p / 2} \frac{1}{p} \cot \left[\frac{\pi\left(x-x^{\prime}\right)}{p}\right] \frac{d U\left(x^{\prime}\right)}{d x^{\prime}} d x^{\prime} \\
& =\frac{\tau}{2 \pi} \sin [2 \pi U(x) / c],
\end{aligned}
$$

where boundary conditions are $U(-p / 2)=0$ and $U(p / 2)$ $=c$. If we use the notation

$$
\begin{gathered}
X=\tan \frac{\pi x}{p} \\
X^{\prime}=\tan \frac{\pi x^{\prime}}{p} .
\end{gathered}
$$

Equation (9) can be written as

$$
-\frac{\mu}{p} \int_{-\infty}^{+\infty} \frac{1+X X^{\prime}}{X-X^{\prime}} d U\left(X^{\prime}\right)=\frac{\tau}{2 \pi} \sin [2 \pi U(X) / c] .
$$

In general, there is no systematic method to solve the integro-differential Eq. (11). However, similar to original PN model, we guess that Eq. (11) has the following analytic solution

$$
U(X)=\frac{c}{2}+\frac{c}{\pi} \arctan [g(\beta) X]
$$




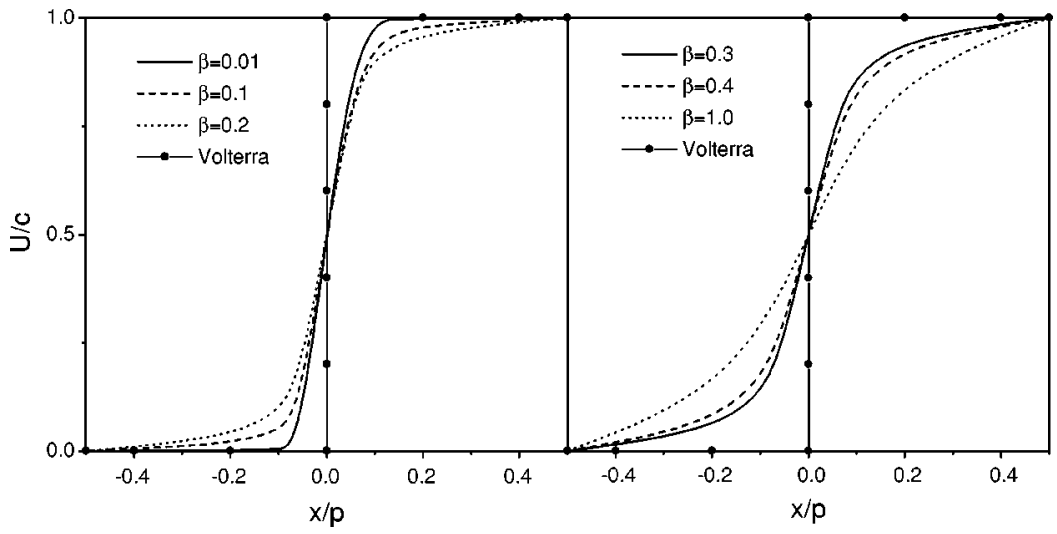

FIG. 2. Relative displacement $U(x)$ for various $\beta . U(x)$ of the singular-Volterra dislocation is also shown for comparison. All the quantities are dimensionless.

where

$$
\begin{gathered}
g(\beta)=\sqrt{\beta^{-2}+1}+\beta^{-1} \\
\beta=\frac{2 \pi c \mu}{p \tau}=\frac{2 \pi \mu f}{\tau} .
\end{gathered}
$$

Substituting Eq. (12) into Eq. (11), this solution can be easily verified. Here, the dimensionless number $\beta$ is a key physical parameter, which controls the structure of interface, it decreases with increasing of $\tau$ and decreasing of $f$. The importance of $\beta$ will be shown in Sec. III. By inserting Eq. (10) into Eq. (12), we can obtain

$$
U(x)=\frac{c}{2}+\frac{c}{\pi} \arctan \left[\left(\sqrt{\beta^{-2}+1}+\beta^{-1}\right) \tan \frac{\pi x}{p}\right]
$$

and plastic displacement

$$
u(x)=\frac{c}{\pi} \arctan \left[\left(\sqrt{\beta^{-2}+1}+\beta^{-1}\right) \tan \frac{\pi x}{p}\right]-\frac{c}{p} x .
$$

A graph of $U(x)$ is shown in Fig. 2, and it shows that $U(-p / 2)=0$ and $U(p / 2)=c$. Also, $U(-\zeta / 2)=c / 4$ and $U(\zeta / 2)=3 c / 4$. Thus, the half width of the misfit dislocation core can be defined as

$$
\zeta=\frac{2 p}{\pi} \arctan \left[\beta\left(\sqrt{\beta^{2}+1}-\beta\right)\right] .
$$

Clearly, the misfit dislocation core width $\zeta$ increases as $\beta$ increases. This illustrates the fact that misfit $f$ and bond strength $\tau$ can influence the structure of the misfit dislocation. The Eq. (16) shows that an increase in bond strength $\tau$ leads to decreasing in width of misfit dislocation core, whereas at constant bond strength $\tau$, the width of misfit dislocation increases with decreasing of misfit $f$.

Through Fourier analysis, a solution similar to Eq. (14) had been obtained with neglecting the normal forces by Merwe. ${ }^{11,12}$ Merwe's result is an approximate solution, and in which normal displacement is not continuous. In our derivation, the continuity of tractions and displacements at interface is assured, since Eq. (3) is the exact elastic stress solution of an edge dislocation at interface. The difference between the two solutions is that $\mu=1 /\left[\left(1-\nu_{1}\right) / \mu_{1}+(1\right.$ $\left.\left.-\nu_{2}\right) / \mu_{2}\right]$ in Merwe's method, while in our method $\mu$ $=\left[2 \mu_{2} /\left(\kappa_{2}+1\right)\right]\left[\left(1+\alpha_{1}\right) /\left(1-\alpha_{2}^{2}\right)\right]$. Apparently they are equal only when the two components of interface have same elastic parameters; otherwise, they are not equal, and the relative difference between them may be up to $13 \%$.

\section{DISCUSSION AND APPLICATION}

\section{A. Energy of misfit dislocation}

Similar to the original PN model, energy of the misfit dislocation is calculated as the sum of two contributions: the elastic strain energy stored in the two half crystals and the misfit energy associated with the nonlinear distortion of bonds across the slip plane. ${ }^{19}$ The former can be written as

$$
E_{d i s l}^{e l}(R)=-\frac{1}{2} \int_{-R}^{+R} \sigma_{x y}(x) u(x) d x,
$$

where $R \leqslant p / 2 . E_{\text {disl }}^{e l}(R)$ is the elastic energy contributed by the stresses within the interval $-R$ to $R$ at interface. With $u(x)$ described by Eq. (15), the shear stress $\sigma_{x y}$ along interface is

$$
\sigma_{x y}(x)=-\frac{\tau}{2 \pi} \frac{\beta \sin \left(\frac{2 \pi x}{p}\right)}{\sqrt{\beta^{2}+1}-\cos \left(\frac{2 \pi x}{p}\right)} .
$$

Then, inserting Eqs. (18) and (15) into Eq. (17), one obtains

$$
\begin{gathered}
E_{d i s l}^{e l}(R)=\frac{\mu c^{2}}{2 \pi} w(R / p) \\
w(r)=\int_{-r}^{r} \frac{\sin (2 \pi x)\{\arctan [g(\beta) \tan (\pi x)]-\pi x\}}{\sqrt{\beta^{2}+1}-\cos (2 \pi x)} d x .
\end{gathered}
$$

For singular-Volterra-type dislocation, the elastic selfenergy per unit length along the axis of a cylinder of radius $R$ around the dislocation can be written as

$$
E_{d i s}=E_{c o r e}+K \ln \frac{R}{r_{c}}
$$




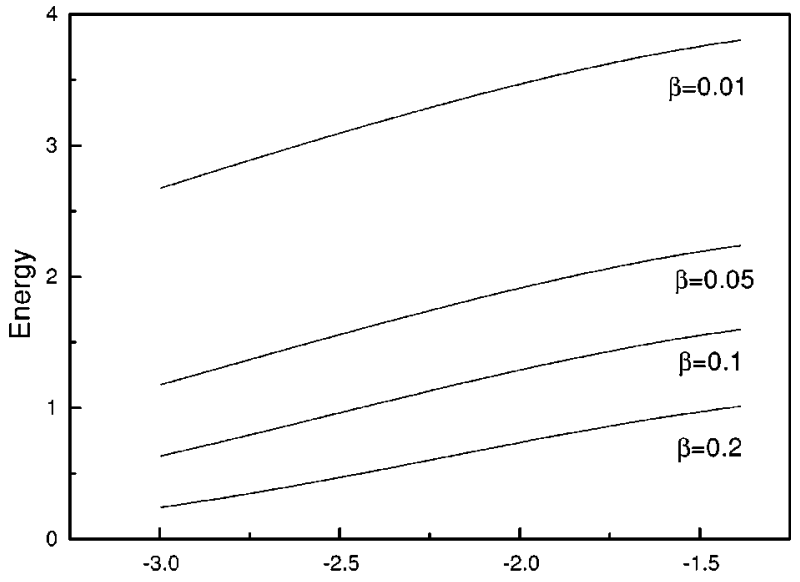

(a)

$\ln R / p$

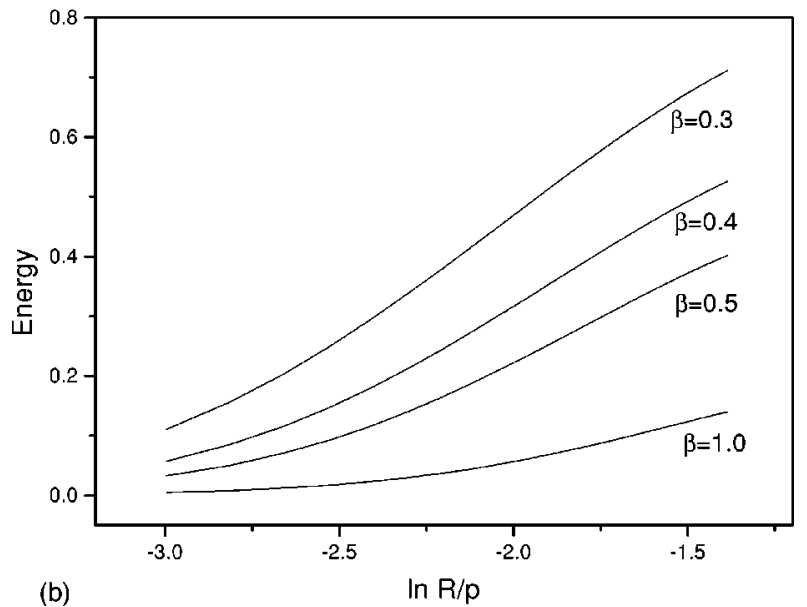

FIG. 3. Elastic energy $E_{d i s l}^{e l}$ vs $\ln (R / p)$ for various $\beta$. Energy is in units of $\mu c^{2} / 2 \pi$. $R$ represents the distance from the center of the dislocation at interface and $p$ is the period of misfit dislocation array.

where $r_{c}$ represents the core radius, and energy factor $K$, which is introduced by Foreman, ${ }^{20}$ is constant related to materials' parameter. If elastic energy of the misfit dislocation is also linearly correlated with $\ln R$, we can say the misfit dislocation is like singular-Volterra-type dislocation, and the structure of the interface can be qualified as an array of singular-Volterra-type interface dislocations. In the following, we compute the elastic energy of dislocations as a function of distance to the center.

Figure 3 illustrates that the elastic energies $E_{\text {disl }}^{e l}$ increases linearly with $\ln (R)$ for interface with small $\beta$, but not with large $\beta .^{21}$ This transition occurs when $\beta$ is about $0.2-0.4$. This shows that the MD structure for $\beta<0.2$ is dislocationlike, i.e., in terms of a singular-Volterra type. The viewpoint can also be confirmed by Fig. 2, and it shows that $U(x)$ is more closer to singular-Volterra dislocation for smaller $\beta$. The width of misfit dislocation core $\zeta / p$ is bigger than $10 \%$ when $\beta>0.2$, in which case the core part of misfit dislocation cannot be neglected compared with the whole interface, so here it is unreasonable to describe the interface structure as an array of singular-Volterra dislocations. This means that low-misfit and high-interfacial bond strength is favorable for nucleating dislocation-like local structure, and only interface with small $\beta$ can be represented by an array of singular-
Volterra dislocation. And this is qualitatively agreed to that of atomic simulation by W. P. Vellinga. ${ }^{8}$ For the case of $\beta$ $<0.2$, the energy factor $K$ of misfit dislocation is in range of $0.5-0.7\left(\mu c^{2} / 2 \pi\right)$.

The total misfit energy can be written as

$$
E_{\text {disl }}^{\text {misift }}=\int_{-p / 2}^{+p / 2} \Phi[U(x)] d x
$$

with the approximation in Eq. (8), $\Phi(U)$ is defined as $\Phi(U)=-\int F(U) d U=\tau c\{1-\cos [2 \pi U(x) / c]\} / 4 \pi^{2}$. Integrating Eq. (21) with the help of Eq. (14), one can obtain

$$
E_{\text {disl }}^{\text {misfit }}=\frac{\mu c^{2}}{2 \pi} \frac{1+\beta-\sqrt{1+\beta^{2}}}{\beta} .
$$

Equation (22) shows misfit energy increases with decreasing of $\beta$. At constant $\tau$, the smaller misfit the larger misfit energy, and this bears out the results of atomic simulation. ${ }^{8}$

\section{B. Interfacial energy and adhesive work}

The mean total energy per unit area of interface due to the forming of misfit dislocation is called interfacial energy and can be written as

$$
E=\frac{1}{p}\left[E_{\text {disl }}^{e l}(p / 2)+E_{\text {disl }}^{\text {misift }}\right] .
$$

Integrating Eq. (20), we can give $w(1 / 2)=$ $-\ln \left[2 \beta \sqrt{1+\beta^{2}}-2 \beta^{2}\right]$, then the interfacial energy can be written as

$$
E=\frac{\tau c}{4 \pi^{2}}\left(1+\beta-\sqrt{1+\beta^{2}}-\beta \ln \left[2 \beta \sqrt{1+\beta^{2}}-2 \beta^{2}\right]\right) .
$$

Expression (24) means that to estimate interfacial energy need to know magnitude of $\tau$. In original PN model, $\tau$ can be chosen so that, for small displacements, the elastic limit is recovered. However, given interface, it is not easy to estimate $\tau$ by experiment. One effective approach is to obtained it by $a b$ initio electronic calculation. Schönberger et al. ${ }^{22}$ made $a b$ initio electronic calculations for $\mathrm{Ag} /\{100\} \mathrm{MgO}$ and $\mathrm{Ti} /\{100\} \mathrm{MgO}$ interfacial system, and they presented the force constants parallel to the interface $0.5 \mathrm{eV} / \mathrm{Ag} / \mathrm{MgO} / \AA^{2}$ and $1.7 \mathrm{eV} / \mathrm{Ti} / \mathrm{MgO} / \AA^{2}$, respectively. Then, the corresponding bond strength $\tau$ can be calculated and they are 3.86 $\times 10^{10} \mathrm{~Pa}$ for $\mathrm{Ag} / \mathrm{MgO}$ and $13.05 \times 10^{10} \mathrm{~Pa}$ for $\mathrm{Ti} / \mathrm{MgO}$, respectively. Dimensionless numbers $\beta$ are 0.185 for $\mathrm{Ag} / \mathrm{MgO}$ and 0.042 for $\mathrm{Ti} / \mathrm{MgO}$, respectively, and both are smaller than 0.2 . These show that $\mathrm{Ag} /\{100\} \mathrm{MgO}$ and $\mathrm{Ti} /\{100\} \mathrm{MgO}$ interface can be represented by an array of singular-Volterra dislocation.

The theoretical adhesive work by ab initio calculation exceeds the experimental estimate because in the experimental sample there are misfit dislocations, whose energy reduces the adhesion. ${ }^{23}$ However, the energy of misfit dislocations cannot be directly calculated since the unit cells, which are required including one misfit dislocation, are too large for $a b$ initio method. To obtain the sound value, the theoretical adhesive work by ab initio calculation must exclude interfacial 
energy due to the forming of misfit dislocation. Assuming that the interface is constructed of a square network of misfit dislocation with Burgers vectors of the $c\langle 100\rangle$ type, Eq. (24) gives interfacial energy $313.4\left(\mathrm{~mJ} / \mathrm{m}^{2}\right)$ for $\mathrm{Ag} /\{100\} \mathrm{MgO}$ and $408.4\left(\mathrm{~mJ} / \mathrm{m}^{2}\right)$ for $\mathrm{Ti} /\{100\} \mathrm{MgO}$, respectively. Therefore, for $\mathrm{Ag} /\{100\} \mathrm{MgO}$ interface, the theoretical adhesive work will be reduced from $900\left(\mathrm{~mJ} / \mathrm{m}^{2}\right)$ to $586.6\left(\mathrm{~mJ} / \mathrm{m}^{2}\right)$, and is a bit larger than experiment value 450 $\pm 100\left(\mathrm{~mJ} / \mathrm{m}^{2}\right) .{ }^{23} \mathrm{We}$ believe that the error remained is due to sinusoidal approximation of the restoring force $F[U(x)]$. In order to gain more precise adhesive work, the exact restoring force $F[U(x)]$, which is obtained by ab initio calculation, will be put to use in the future work.

\section{SUMMARY AND CONCLUSIONS}

Following the original Peierls-Nabarro's idea, PN model of periodic misfit dislocation array is developed in the paper, and is exactly analytically solved. The extended model can clarify the effects of different bonding strengths and misfit on the interface structure. The solution shows that the core structure and the core energy of misfit dislocation clearly depend on bond strength and misfit parallel to the interface. The dimensionless number $\beta=2 \pi \mu f / \tau$ determines the structure and the energy of interface, and only when $\beta$ $<0.2$ the structure of interface can be represented by an array of singular-Volterra dislocations.

Obviously, our method provides a correlation between $a b$ initio calculations and the continuum theory. It enables us to estimate interfacial energy and adhesive work simply by inputting ab initio calculation data. For example, we can determine that the adhesive work of $\mathrm{Ag} /\{100\} \mathrm{MgO}$ interface is about $586.6\left(\mathrm{~mJ} / \mathrm{m}^{2}\right)$.

Finally, our approach can be easily applied to the interface, which is constructed of periodic screw dislocation array.
*Electronic address: tcwang@cc5.imech.ac.cn

${ }^{1}$ J. P. Hirth and X. Feng, J. Appl. Phys. 67, 3343 (1992); X. Feng, and J. P. Hirth, ibid. 72, 1386 (1992); H. O. K. Kirchner, Phys. Scr. 44, 151 (1992).

${ }^{2}$ F. C. Frank and J. H. van der Merwe, Proc. R. Soc. London, Ser. A 198, 205 (1949); 198, 216 (1949).

${ }^{3}$ J. W. Matthews, in Dislocations in Solids, edited by F. R. N. Nabarro (North-Holland, Amsterdam, 1979), Vol. 2, p. 461.

${ }^{4}$ Y. Ishida, J.-Y. Wang, T. Suga, and S. Tanaka, in Metal-Ceramic Interfaces, edited by M. Rühle, A. G. Evans, M. F. Ashby, and J. P. Hirth (Pergamon Press, Oxford, 1990), p. 234; F. S. Shien and S. L. Sass, in Metal-Ceramic Interfaces, ibid., p. 258; D. Knauss and W. Mader, Ultramicroscopy 37, 247 (1991); S. Ernst, P. Pirouz, and A. H. Heuer, Philos. Mag. A 63, 259 (1991); D. X. Li, P. Pirouz, A. H. Heuer, S. Yadavalli, and C. P. Flynn, Acta Metall. Mater. 40, S237 (1992); K. L. Merkle, M. I. Buckett, and Y. Gao, ibid. 40, S249 (1992); A. Trampert, F. Ernst, C. P. Flynn, H. Fischmeister, and M. Rühle, ibid. 40, S227 (1992); P. Lu and F. Cosandey, ibid. 40, S259 (1992); J. Bruley, R. Brydson, H. Mullejans, J. Mayer, G. Gutekunst, W. Mader, D. Knauss, and M. F. Rühle, J. Mater. Res. 9, 2574 (1994); H. Jang, D. N. Seidman, and K. L. Merkle, Interface Sci. 1, 61 (1993).

${ }^{5}$ V. Vitek, Philos. Mag. A 71, 1219 (1995).

${ }^{6}$ S. C. Jain, J. R. Willis, and R. Bullough, Adv. Phys. 39, 127 (1990); S. V. Kamat and J. P. Hirth, J. Appl. Phys. 67, 6844 (1990); J. R. Willis, S. C. Jain, and R. Bullough, Philos. Mag. A 62, 115 (1990); 64, 629 (1991); K. Jagannadham and J. Narayan, Mater. Sci. Eng., B 8, 107 (1991); A. Atkinson and S. C. Jain, J. Phys.: Condens. Matter 5, 4595 (1993); T. J. Gosling, F. R. Willis, R. Bullough, and S. C. Jain, J. Appl. Phys. 73, 8297 (1993); W. A. Jesser and J. M. Kui, Mater. Sci. Eng., A 164, 101 (1993); G. E. Beltz and L. B. Freund, Philos. Mag. A 69, 183 (1994).

${ }^{7}$ A. S. Nandekar and J. Narayan, Philos. Mag. A 61, 873 (1990); P. Gumbsch, M. S. Daw, S. M. Foiles, and H. F. Fischmeister,
Phys. Rev. B 43, 13833 (1991); A. S. Nandekar, G. R. Srinivasan, and C. S. Murthy, ibid. 43, 7308 (1991); A. S. Nanderkar, Acta Metall. Mater. 41, 3455 (1993).

${ }^{8}$ W. P. Vellinga, J. Th. M. De Hosson, and V. Vitek, Acta Mater. 45, 1525 (1997).

${ }^{9}$ R. Peierls, Proc. Phys. Soc. London 52, 34 (1940); F. R. N. Nabarro, ibid. 59, 256 (1947).

${ }^{10}$ B. Joós and M. S. Duesbery, Phys. Rev. Lett. 78, 266 (1997).

${ }^{11}$ J. H. Van der Merwe, Proc. Phys. Soc. London, Sect. A 63, 616 (1950).

${ }^{12}$ J. H. Van der Merwe, J. Appl. Phys. 34, 117 (1963).

${ }^{13}$ J. H. Van der Merwe and W. A. Jesser, J. Appl. Phys. 63, 1509 (1988).

${ }^{14} \alpha_{1}=\left[\Gamma\left(\kappa_{2}+1\right)-\left(\kappa_{1}+1\right)\right] /\left[\Gamma\left(\kappa_{2}+1\right)+\left(\kappa_{1}+1\right)\right], \quad \alpha_{2}=\left[\Gamma\left(\kappa_{2}\right.\right.$ $\left.-1)-\left(\kappa_{1}-1\right)\right] /\left[\Gamma\left(\kappa_{2}+1\right)+\left(\kappa_{1}+1\right)\right]$, and $\Gamma=\mu_{1} / \mu_{2}, \kappa_{1}=3$ $-4 \nu_{1}, \kappa_{2}=3-4 \nu_{2}$ for plane strain. The derivation of expression (3) can refer to Z. Suo and J. W. Hutchinson, Int. J. Fract. 43, 1 (1990).

${ }^{15}$ J. P. Hirth and J. Lothe, Theory of Dislocations, 2nd ed. (Wiley, New York, 1982), p. 732.

${ }^{16}$ V. Vitek, Philos. Mag. 18, 773 (1968).

${ }^{17}$ E. Kaxiras and M. S. Duesbery, Phys. Rev. Lett. 70, 3752 (1993).

${ }^{18}$ B. Joós, Q. Ren, and M. S. Duesbery, Phys. Rev. B 50, 5890 (1994); 53, 11 882(E) (1996); Q. Ren, B. Joós, and M. S. Duesbery, ibid. 52, 13223 (1995); 53, 11 883(E) (1996).

${ }^{19}$ J. P. Hirth and J. Lothe, Theory of Dislocations (Ref. 15), pp. 226-237.

${ }^{20}$ A. J. E. Foreman, Acta Metall. 3, 322 (1995).

${ }^{21}$ The linear behavior of $E_{\text {disl }}^{e l}$ vs $\ln (R / p)$ must break down in all cases as $R$ approaches $p / 2$ due to the interaction of dislocations. Here, we choose $R$ in range of $(0, p / 4)$, because $E_{d i s l}^{e l}(p / 4)$ occupies more than $90 \%$ of total elastic energy $\left[E_{d i s l}^{e l}(p / 2)\right]$ when $\beta<0.2$.

${ }^{22}$ U. Schönberger, O. K. Andersen, and M. Methfessel, Acta Metall. Mater. 40, S1 (1992).

${ }^{23}$ M. W. Finnis, J. Phys.: Condens. Matter 8, 5811 (1996). 